\title{
Conversión del dominio discreto en dominio continuo: Otro enfoque de aprendizaje
}

The domain discreet in continuous domain conversion: another approach to learning

\author{
Kemel George González \\ Doctorado en Matemática, Centro De Investigación Y De Estudios Avanzados Del IPN, Director Maestrría en Educación y \\ Director de Regalías de la Universidad Autónoma del Caribe, Kemel.george@gmail.com \\ Para Citar este Artículo: George, Kemel (2017), 15 (1), p,p 160-168. \\ DOI: http:/ / dx.doi.org/10.15665/esc.v15i1.1172
}

Recibido: Abril 3 de 2017

Aceptado: Abril 28 de 2017

\begin{abstract}
(n)
\section{RESUMEN}

En la teoría de la comunicación, la señal, como ente portador de información, se modela matemáticamente como una colección finita de valores numéricos en el dominio temporal y en el dominio frecuencial. Ambos dominios son discretos finitos y se distinguen entre sí, porque unos valores del fenómeno representado en un dominio se intercambian o transforman en valores del otro dominio, mediante la denominada Transformada Discreta de Fourier. Acorde a los conceptos convencionales del aprendizaje, es usual concentrarse más en la representación del fenómeno mismo, que en el dominio en el cual se representa. Se plantea en este artículo una reflexión derivada de un trabajo doctoral, que el proceso de aprendizaje debería ser el inverso, ya que el estudio del dominio de la señal y su conversión del discreto al continuo proporciona, como en efecto lo veremos, una valiosa información sobre la naturaleza de la relación entre la conexión de los valores temporales y frecuenciales y su íntimo vinculo con la teoría de Fourier, siempre que el campo numérico de representación se extienda a cantidades finitas, infinitas e infinitesimales.
\end{abstract}

Palabras clave: dominio temporal, frecuencial, transformación, Análisis de Fourer.

\begin{abstract}
In communication theory, signal, as a carrier of information, is mathematically modelled as a finite collection of numerical values in the temporal domain and the frequency domain. Both domains are finite discrete and are distinguished among themselves, because the phenomenon depicted in a domain values are exchanged or transformed into values of the other domain, using the so-called discrete transformed of Fourier. According to the conventional concepts of learning, it is usual to concentrate more on the representation of the same phenomenon, which in the domain in which it is represented. Arises in this article a reflection derived from doctoral work, that the learning process should be the inverse, since the study of the domain of the signal and its conversion of the discrete to the continuous provides, as we shall indeed see it, valuable information on the nature of the relationship between temporal and frequency values connection and its intimate link...
\end{abstract}

Key words: time, frequency, domain transformation, analysis of Fourer 


\section{El dominio discreto temporal}

Es usual que, en la representación funcional de la señal, donde el número de muestras o valores es finito, apenas se le preste atención al dominio, limitándose su descripción a la selección de $\mathrm{N}$ puntos, digamos $\left\{C_{n}\right\}$ donde el índice recorre los números naturales cuando se calculan los coeficientes de Fourier, acorde a Pinsky, M. A. (2003) o subíndices como $A_{r}, X_{k}$ donde los índice recorren un conjunto finito, cuando se calcula la Transformada Discreta de Fourier Cochran, W. C. Cooley, J. W. et al ( 1967 ). Y en efecto, esta simplificación es conveniente ya que se trata de resaltar la representación del fenómeno y no tanto, del dominio numérico en que se desenvuelve. No ocurre así cuando hay múltiples reresentaciones de la señal y una interdependencia que requiere de manera muy precisa, la determinación de cada uno de los parámetros que posibilitan la transformación de valores en un dominio, a valores en otro dominio.

Este es precisamente el caso que queremos dilucidar. Vamos a mostrar que la determinación de las relaciones mutuas entre los dominos temporal y frecuencial propios en los que la señal se representa, brindan información sobre el comportamiento del fenómeno, e indican las reglas de conversión de cantidades discretas finitas a cantidades continuas, una vez que la estructura aritmética no sea la del familiar campo de los números reales, sino de extensiones que contienen cantidades finitas, infinitas e infinitesimales George, K. (1998).

Para ello, es necesario fijar variables cuyas relaciones entre sí, no solo determinan los dominios temporal y frecuencial, sino que indican de modo natural la conversión del discreto al continuo. Para ello, consideremos el número real finito $0<\mathrm{T} 0$ y $\mathrm{N} \in \bullet$ entero positivo; llamaremos dominio temporal al conjunto de $2 \mathrm{~N}$ elementos

$\left\{\frac{n T}{N}\right\}=\left\{-\frac{N T}{N}, \frac{(N-1) T}{N}, \cdots,-\frac{2 T}{N},-\frac{T}{N}, 0, \frac{T}{N}, \frac{2 T}{N}, \cdots, \frac{(N-1) T}{N}\right\}$
En tal dominio, $-N \leq n<N$. Indicaremos cada uno de sus términos como $t_{n}$ y llamaremos $v a-$ riable temporal $t$, a la sucesión,

$$
t=\left\{t_{n}\right\}=\left\{\frac{n T}{N}\right\}
$$

En otras palabras, la variable temporal es una sucesión que recorre cada una de las $t_{n}$ posiciones temporales del dominio considerado, donde $n$ asume $2 \mathrm{~N}$ valores enteros.

Una imagen gráfica del dominio temporal es la siguiente. El segmento de recta [-T, T], se divide en $2 \mathrm{~N}$ intervalos, cada uno de ellos de longitud $\frac{T}{N}$, con $2 N$ posiciones temporales, excluida la última,

$\begin{array}{lllllll} & 1 & 1 & 1 & \\ -T & -n T / N & -T / N & 0 & T / N & n T / N & T\end{array}$

La imagen no es el continuo real, sino los puntos discretos del dominio.

Aunque la figura parece convencional, no lo será tanto, en la medida en que luego modifiquemos la estructura numérica la cual le sirve de dominio.

Algunos autores Bracewell, R. N. (1985). H. J. Weaver,(1983) llaman a la cantidad 2T, la $d u-$ ración de la señal cuando sus valores están representados en tal intervalo. Aquí se llamará el período del intervalo. En su momento veremos que el calificativo temporal, o espacial u otro cualquiera, depende del fenómeno representado, de acuerdo a que este se encuentre modelado en el tiempo, en el espacio, o en cualquier otra entidad susceptible de cuantificarse y dotarse de unidad de medida. Siguiendo la notación de los ingenieros, nosotros preferiremos la denominación de temporal para el dominio, dado que es unidimensional, pero cuando se considera el dominio bidimensional, el calificativo de espacial, es mucho más conveniente. Físicamente, la representación temporal de la señal está asociada al sonido, al ruido, a la voz humana; mientras que la espacial, a las imágenes, al video y a las gráficas.

Según la convención, en el dominio temporal, $\frac{T}{N}$ corresponde a la primera posición en el tiem- 
po positivo; $\frac{2 T}{N}$ a la segunda, etc. Y así para las posiciones negativas. Ya veremos que, por razones de conveniencia, la última posición $T$ está excluida. A veces la variable $t$ está comprendida en el rango $0 \leq n<N$. En este caso, que no difiere del nuestro, cada dominio tendrá $N$ muestras. Nosotros, siguiendo la tradición en la igeniería, hemos introducido los dominios con posiciones negativas, positivas y cero; aunque la notación se hace más engorrosa, hay una ventaja teórica y didáctica de esta nomenclatura.

El número real $\frac{T}{N}$ siempre designa una fracción de la unidad de la entidad considerada; si ésta es el tiempo, $\frac{T}{N}$ está medida en fracciones de segundo; si es el espacio bidimencional, es una fracción de $\mathrm{cm}^{2}$, etc. Por razones físicas, a la cantidad $\frac{T}{N}$ se le denomina intervalo de muestreo, dado que el fenómeno que se represente asume en el dominio una colección de observaciones o muestras. Algunos llaman el intervalo de muestreo resolución en tiempo, aunque esta denominación es menos frecuente. Por ejemplo, en el reconocimiento de voz, se usa representar la señal con 16.000 muestras cada segundo. En este caso, conviene hacer $2 \mathrm{~T}=1$ segundo, y $\mathrm{N}=$ 8.000. El intervalo de muestreo o resolución en tiempo es $1 / 16.000$ segundos.

\section{El dominio discreto frecuencial}

Todo dominio temporal conlleva otro dominio indisolublemente ligado a él, del siguiente modo. Sea $t=\left\{t_{n}\right\}=\left\{\frac{n T}{N}\right\}$ la variable del dominio temporal. Como $2 T$ cubre la totalidad de las $2 N$ muestras, desde el punto de vista aritmético, su inverso $\frac{1}{2 T}$ es «el número de veces que $2 \mathrm{~T}$ cabe en la unidad». Así, $\frac{1}{2 T}$ se convierte en una fracción de la nueva unidad de medida, universalmente denominada hertz. Como hay $2 \mathrm{~N}$ muestras temporales, la fracción $\frac{1}{2 T}$ genera el dominio de $2 \mathrm{~N}$ elementos $\left\{\frac{k}{2 T}\right\}=\left\{-\frac{N}{2 T},-\frac{N-1}{2 T}, \cdots,-\frac{2}{2 T},-\frac{1}{2 T}, 0, \frac{1}{2 T}, \frac{2}{2 T}, \cdots, \frac{N-1}{2 T}\right\}$

Nuevamente, $-N \leq k<N$.

Este se denomina dominio frecuencial y, por la forma como lo hemos construido, está totalmente determinado por el dominio temporal, siendo esta una relación recíproca. De la misma forma que la variable temporal recorre su dominoo, dicho dominio está recorrido por la variable frecuencial $f$ como sucesión de $2 N$ valores,

$$
f=\left\{f_{k}\right\}=\left\{\frac{k}{2 T}\right\}
$$

La gráfica correspondiente del dominio frecuencial es

\begin{tabular}{lllll}
\hline & 1 & 1 & $\mathrm{kN} / 2 \mathrm{~T}$ & $\mathrm{~N} / 2 \mathrm{~T}$
\end{tabular}

Se dice que $f_{k}$ es la altura de la frecuencia $f$. En resumen, siempre que tengamos $0<T y N$ entero positivo, existe una relación recíproca que determina la construcción de dominios asociados,

$$
\left\{\frac{n T}{N}\right\} \Leftrightarrow\left\{\frac{k}{2 T}\right\}
$$

Así, por ejemplo, sea $T=\frac{3}{2}$ y $N=100$. Inmediatamente, tenemos dos dominios de 200 elementos,

$$
\left\{\frac{3 n}{200}\right\} \Leftrightarrow\left\{\frac{k}{3}\right\} .
$$

El primero es el dominio del tiempo, y el segundo, el dominio de la frecuencia. Cuando $n=2$ $\mathrm{y} \mathrm{k}=99$ hay una posición temporal en $-\frac{3}{100} \mathrm{y}$ una altura de frecuencia 33. Las variables correspondientes están correlacionadas mediante la relación $\quad t_{n}=\frac{n T}{N}, \quad f_{k}=\frac{k}{2 T}$

Reemplazando una en la otra, la relación entre el tiempo y la frecuencia, está dada por la fórmula,

$$
t_{n}=\frac{n k}{2 N} \frac{1}{f_{k}}
$$

Siempre que la frecuencia no esté a la altura cero. Esta y no otra, es la verdadera relación inversa entre la frecuencia y el tiempo en el dominio discreto.

En un dominio como el de arriba, sea $T=\frac{3}{2}$ y $N=100$. La relación entre la posición octava 
negativa temporal y la altura onceava positiva frecuencial es $t_{-8}=\frac{-8 \times 11}{200} \frac{1}{f_{11}}=-0.44 \frac{1}{f_{11}}$. Esta relación no depende del tamaño del intervalo $T$. En ingeniería, a la cantidad $\frac{N}{2 T}$ se la llama ancho de banda Weaver, H. J. (1983) y corresponde a la frecuencia más alta que alcanza la variable $f$. La primera posición frecuencial $\frac{1}{2 T}$ se llama frecuencia fundamental $f_{1}$. Ambas cantidades se miden en hertz. Por ejemplo, para 200 posiciones temporales durante cinco segundos, el intervalo de muestreo será 0.05 segundos, mientras que la frecuencia fundamental es de 0.1 hertz y el ancho de banda será de 10 hertz.

Veamos otro ejemplo. Se requiere de un intervalo de muestreo de una centésima de segundo con 4 posiciones temporales. Por tanto, $N=2$, $\frac{T}{N}=\frac{1}{100},-2 \leq n \leq 1$ y el dominio temporal es $\left\{-\frac{1}{50},-\frac{1}{100}, 0, \frac{1}{100}\right\}$. La frecuencia fundamental es $1 / 2 \mathrm{~T}=25$ hertz. El ancho de banda es 50 hertz. El dominio frecuencial es $\{-50,-25,0,25\}$. El par de dominios asociados, donde $-2 \leq n, k \leq 1$, es

$$
\left\{-\frac{1}{50},-\frac{1}{100}, 0, \frac{1}{100}\right\} \Leftrightarrow\{-50,-25,0,25\}
$$

El siguiente es un caso simple y extremo. Ocurre cuando $2 N=2$. Dado que en el intervalo de muestreo $T=N$ y la frecuencia fundamental es $\frac{1}{2 T}=\frac{1}{2}$; obtenemos los dominios

Un dominio de uso frecuente en ingeniería se obtiene cuando el intervalo de muestreo es la unidad, o sea $T=N$. Es el que podemos leer en todos los libros de texto. Como el intervalo de muestreo es un segundo, la frecuencia fundamental es $\frac{1}{2 N}$ hertz y el ancho de banda es $1 / 2$. Losdominios asociados son,

$$
\{n\} \Leftrightarrow\left\{\frac{k}{2 N}\right\}
$$

Con las siguientes instrucciones del software Mathematica, se obtienen dominios temporales $\mathrm{y}$ frecuenciales.

\section{temporal[T_M_]:=N[Table[n \\ $\mathbf{T} / \mathbf{M},\{\mathbf{n},-\mathbf{M}, \mathbf{M}-\mathbf{1}\}], 2]$; \\ frecuencial[T_M_]:=N[Table[k 1/2T,\{k,-M,M-1\}],2];}

Por ejemplo, si $\mathrm{T}=5, \mathrm{M}=40$; obtenemos:

$\operatorname{In}[1]:=$ temporal[ $[5,40]$

Out $[1]=$

$\{-5 .,-4.88,-4.75,-4.63,-4.5,-4.38,-4.25,-$

$4.13,-4 .,-3.88,-3.75,-3.63,-3.5,-3.38,-$

$3.25,-3.13,-3 .,-2.88,-2.75,-2.63,-2.5,-$

$2.38,-2.25,-2.13,-2 .,-1.88,-1.75,-1.63,-$

$1.5,-1.38,-1.25,-1.13,-1 .,-0.875,-0.75,-$

$0.625,-0.5,-0.375,-0.25,-0.125,0,0.125$

$, 0.25,0.375,0.5,0.625,0.75,0.875,1 ., 1.1$

$3,1.25,1.38,1.5,1.63,1.75,1.88,2 ., 2.13$,

$2.25,2.38,2.5,2.63,2.75,2.88,3 ., 3.13,3$.

$25,3.38,3.5,3.63,3.75,3.88,4 ., 4.13,4.25$

$, 4.38,4.5,4.63,4.75,4.88\}$.

$\operatorname{In}[2]:=$

frecuencial $[5,40]$

Out $[2]=$

$\{-4 .,-3.9,-3.8,-3.7,-3.6,-3.5,-3.4,-3.3,-3.2,-$

$3.1,-3 .,-2.9,-2.8,-2.7,-2.6,-2.5,-2.4,-2.3,-2.2,-$

$2.1,-2 .,-1.9,-1.8,-1.7,-1.6,-1.5,-1.4,-1.3,-1.2,-$

$1.1,-1 .,-0.9,-0.8,-0.7,-0.6,-0.5,-0.4,-0.3,-0.2,-$

$0.1,0,0.1,0.2,0.3,0.4,0.5,0.6,0.7,0.8,0.9,1 ., 1.1,1.2,1$

$.3,1.4,1.5,1.6,1.7,1.8,1.9,2 ., 2.1,2.2,2.3,2.4,2.5,2.6,2$.

$7,2.8,2.9,3.3 .1,3.2,3.3,3.4,3.5,3.6,3.7,3.8,3.9\}$

El intervalo de muestreo es de 0.125 segundos, mientras que la resolución en frecuencia es 0.1 hertz y la frecuencia de muestreo es de 4 hertz. Observemos que podemos indagar por cualquier posición, a partir de cualquier altura de frecuencia. Por ejemplo, queremos saber a cuantos segundos se ubica la muestra quinta negativa, dada la frecuencia en la posición 37, cuya altura es de 3.7 hertz. Por la relación tiempo $\times$ frecuencia, tenemos que

$$
t_{-5}=\frac{(-5)(37)}{2 \times 40} \frac{1}{f_{37}}=-0.625
$$

que corresponde al valor buscado.

\section{El modelo tiempo-frecuencia}

Tradicionalmente, las gráficas del dominio discreto temporal y frecuencial se dibujan por se- 
parado; de un lado, el dominio temporal a la izquierda o arriba; de otro lado, el dominio frecuencial a la derecha o abajo, como en la figura. Esta separación conviene para ilustrar que una cosa es el fenómeno descrito en el tiempo y otra es el fenómeno descrito en la frecuencia.

$\stackrel{-5}{r}$

Como ambos dominios están correlacionados, también es conveniente representar el fenómeno de modo simultáneo, tanto en el tiempo, como en la frecuencia, siendo a veces más útil esta última representación que la primera. La figura 1 en el plano, donde se combinan ambos dominios, es como sigue:

Fig. 1 Dominios de doble representación temporal-frecuencial.

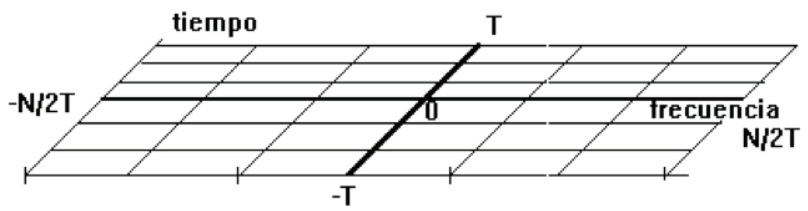

Fuente: el autor.

Obsérvese que hemos fusionado las dos gráficas del dominio de tiempo y la frecuencia, en un sólo dominio bidimensional, que son los ejes tiempo- frecuencia. Así mismo, hemos colocado las posiciones temporales - o espaciales- en el eje vertical, en segundo plano, mientras que las posiciones frecuenciales están en primer plano, en el eje horizontal, para destacar el predominio de la representación en frecuencia. Por una razón visual, se han dibujado trazos continuos, aunque los dominios respectivos están constituidos por los puntos de intersección de las líneas en la gráfica. Ya veremos la utilidad de esta representación cuando se expresan simultaneamente, los valores de las señales en el dominio temporal-frecuencial.

\section{Dominio de alta resolución temporal}

Los términos «resolución en tiempo» $\mathrm{y}$ «resolución en frecuencia» nos indican que es necesario profundizar en la naturaleza de los domi- nios discretos finitos temporales y frecuenciales y de sus relaciones recíprocas. Vamos a partir de la suposición de que sobre tales dominios se representan fenómenos, de los cuales se extraen datos observables o muestras, que de algun modo nos dan alguna información del fenómeno considerado. Admitamos que hemos obtenido una serie de ellas, por ejemplo, $2 \mathrm{~N}$ muestras temporales. ¿Qué modificación se introducen en los dominios si los alteramos con una o más observaciones?

En el dominio frecuencial $\{\mathrm{k} / 2 \mathrm{~T}\}$ tenemos $2 \mathrm{~N}$ posiciones, donde cada una está dada por el índice $k$ que recorre los números enteros - $(\mathrm{N}-1) \mathrm{k}$ $<$ N. ¿Que pasa si aumentamos la frecuncia de muestreo, añadiendo una muestra? Es evidente que el dominio frecuencial se modifica únicamente en el alcance de $\mathrm{k}$, cuyo nuevo rango será $-(\mathrm{N}+1) \mathrm{k}<\mathrm{N}+1$. Al dominio se le añaden dos frecuencias, una a la izquierda y otra a la derecha, que son $f_{-N}, f_{N}$.

No es tan evidente lo que ocurre en el dominio temporal. El período 2T permanece inalterable, o sea que las modificaciones ocurren dentro del intervalo $[-\mathrm{T}, \mathrm{T}]$. Ya que hay $2(\mathrm{~N}+1)$ muestras, el intervalo de muestreo o resolución en tiempo será $\mathrm{T} /(\mathrm{N}+1)$. El nuevo dominio temporal es $\{\mathrm{nT} /(\mathrm{N}+1)\}$. Las $2(\mathrm{~N}+1)$ posiciones temporales se han redistribuído, debido al cambio de la resolución en tiempo.

Esto indica que ambos dominios se desempeñan de modo distinto, a medida que aumenta la frecuencia de muestreo. En el dominio frecuencial, las nuevas posiciones se añaden consecutivamente ya que la última deja inalterable las primeras. En el temporal, las nuevas posiciones se añaden resolutivamente, ya que la última modifica todas las anteriores, manteniendo el dominio fijo.

La consecuencia es inmediata. Si hacemos N muy grande, como $\mathrm{T}$ es fijo, la frecuencia de muestreo $\mathrm{N} / 2 \mathrm{~T}$ se hará muy grande, sin afectar el dominio de la frecuencia; en cambio, la resolución en tiempo $\mathrm{T} / \mathrm{N}$ se hará extremadamente pequeña, para que en el intervalo $[-\mathrm{T}, \mathrm{T}]$ se puedan acomodar $2 \mathrm{~N}$ muestras. Bajo estas condi- 
ciones, el dominio temporal se convierte en un dominio de alta resolución.

Vamos a decribir lo que ocurre en el dominio temporal, cuando la frecuencia de muestreo es extremadamente alta, o sea, cuando el dominio temporal es de alta resolución. Obsérvese que en el dominio temporal, el período $2 \mathrm{~T}$ siempre está fijo y las posiciones de la variable $t=\mathrm{nT} / \mathrm{N}$ van llenando el intervalo [-T, T]. En el dominio frecuencial, las posiciones de la variable $f$ $=\mathrm{k} / 2 \mathrm{~T}$ simplemente se mueven hacia las altas frecuencias. Para simplificar, sólo escribimos la parte positiva de los dominios asociados y dibujamos la parte positiva de la gráfica bidimensional.

\section{Gráfica 1}

Iniciamos con el par $\{0, \mathrm{~T} / 2\}-\{0,1 / \mathrm{T}\}$, donde $\mathrm{N}=2$

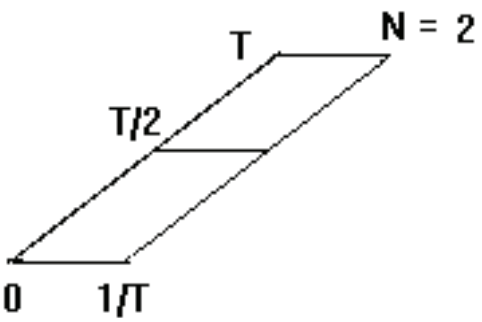

\section{Gráfica 2}

Seguimos con el par $\{0, \mathrm{~T} / 3,2 \mathrm{~T} / 3\}-\{0,1 / \mathrm{T}$, $2 / T$, para $N=3$. El intervalo de muestreo es $\mathrm{T} / 3$.

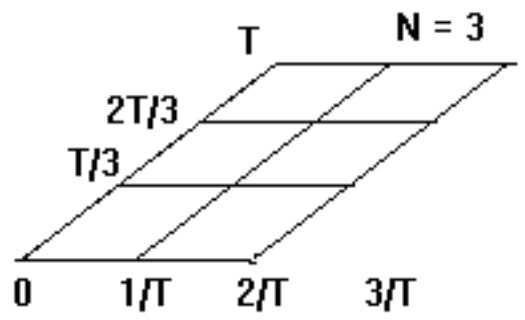

\section{Gráfica 3}

Hacemos $N=5$. Obtenemos el par asociado

$\{0, \mathrm{~T} / 5,2 \mathrm{~T} / 5,3 \mathrm{~T} / 5,4 \mathrm{~T} / 5\}-\{0,1 / \mathrm{T}, 2 / \mathrm{T}, 3 / \mathrm{T}$, $4 / \mathrm{T}\}$
Nótese que T se mantiene fijo.

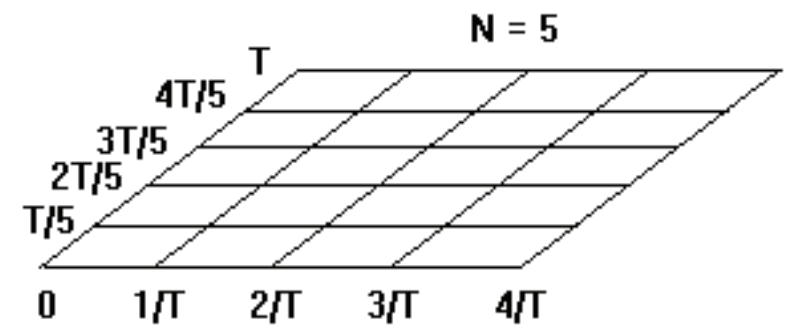

Gráfica 4

Hacemos $\mathrm{N}=9$. Mientras que en el dominio temporal se ha subdividido el intervalo fijo $[0, T]$ en 9 posiciones temporales, las posiciones frecuenciales se han movido hacia la derecha.

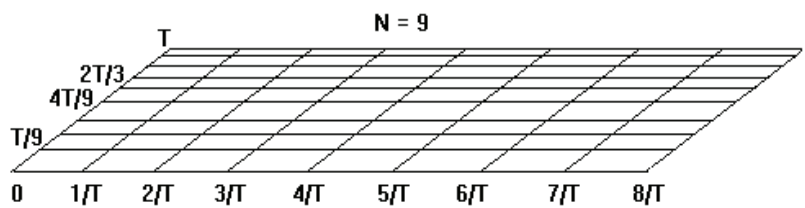

Gráfica 5

Hacemos $\mathrm{N}$ un entero muy grande, por ejemplo, $\mathrm{N}=1000$. El intervalo de muestreo $\mathrm{T} / 256$ es de tamaño inferior a un pixel del monitor del computador. La gráfica del computador sugiere que, en el dominio temporal, se ha "llenado" el intervalo "continuo" [0, T]. Esto es solo una metáfora. Sabemos que no puede llegarse al continuo mediante particiones del intervalo discreto, así estas tiendan a un límite, porque los valores se mantienen en el campo de los números números reales.

Por el lado del dominio frecuencial, la variable $f$ recorre un número muy grande de posiciones $\mathrm{k} / \mathrm{T}$, y ocurre un fenómeno similar, que se mantiene en el dominio discreto.

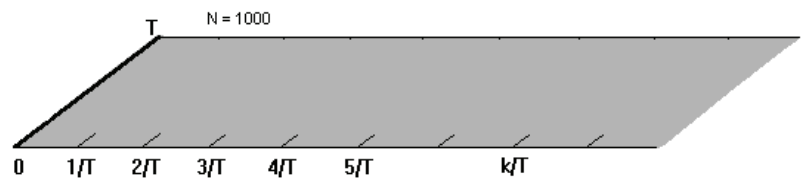

Este tipo de representación, que hemos llamado alta resolución temporal, aunque en ambos casos sigue siendo propia del dominio discreto, es el paso previo al dominio continuo, como lo veremos enseguida. 


\section{Cantidades finitas, infinitas e infinitesimales}

Este es el momento de resolver el problema planteado, que es la posibilidad de convertir el dominio continuo temporal en un paso mucho más allá del dominio discreto de alta resolución, que es el dominio continuo, algo que es insoluble en el cálculo tradicional. Básicamente, se trata de hacer de la resolución temporal, una cantidad infinitesimal.

Vamos a considerar cantidades muy usadas en el cálculo no convencional: son las cantidades infinitas e infinitesimales. Es el momento de introducirlas de modo claro y preciso, se trata de asumir el sistema numérico denominado campo hiperreal o sistema de números hiperreales Robinson, A, Non-Standard (1966), revisada Luxemburg, W.A.J (1962) *o . Este es un campo de propiedades excepcionales en relación al familiar sistema de números reales ${ }^{\circ}$. El campo real está contenido en el campo hiperreal, - $\subset * 0$, y tanto el segundo, como el primero, son un sistema numérico totalmente ordenado.

Mientras que los elementos $r \in \mathbf{R}$ se llaman reales ordinarios, o simplemente números reales, los elementos $* r \in *^{* \circ}$ se denominan números hiperreales o cantidades hiperreales. De la estructura numérica y lógica del sistema hiperreal se desprende que hay tres tipos de números hiperreales, o cantidades:

1. Infinitesimales (o infinitamente pequeños), y se denominan así porque, en valor absoluto, son menores que cualquier número real ordinario. El cero es el único real infinitesimal.

2. Finitos, que son los hiperreales comprendidos entre dos números reales ordinarios. Puede probarse que todo hiperreal finito se descompone de modo único como suma de un número real (su parte estándar) y un infinitesimal Luxemburg, W.A.J (1962).

3. El tercer tipo de hiperreales son los hiperreales infinitos (o infinitamente grandes), que son los hiperreales que en valor absoluto son mayores que todo número real. Un caso particular lo constituyen los enteros infini- tos, llamados hiperenteros $N$. Como puede verse, los hiperreales se particionan en tres clases numéricas determinadas y separadas entre sí: infinitesimales, finitos e infinitos.

\section{El continuo temporal}

El dominio continuo temporal surge cuando se fija un número real finito $\mathrm{T}>0$, y se escoge un entero infinito $N$, esto es, un número entero positivo mayor que cualquier otro entero finito. Sea $-N \leq n<N$. Hacemos $d t$ la cantidad, $d t=\frac{T}{N}$

A esta cantidad se le denomina diferencial de la variable t. Podemos esclarecer totalmente lo que ocurre con la colección $\{n d t\}$ contenida en el intervalo $[-T, T]$. Como el numerador $\mathrm{T}$ es finito y el denominador $N$ es entero infinito, necesariamente la cantidad $d t$ es infinitesimal, esto es, un número positivo menor que cualquier número real. Esta colección se puede describir gráficamente de la siguiente manera,

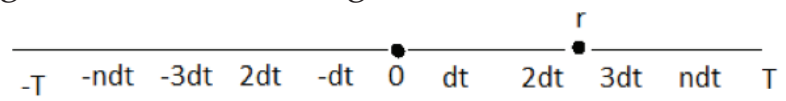

Donde hemos señalado con la letra $-T \leq r<T$ cualquier número real ordinario del intervalo $[-T, T]$. Existe un múltiplo $\mathrm{n}$ del infinitesimal $d t$ que está infinitamente cerca de $\mathrm{r}$, y este múltiplo n puede ser escogido de modo tal que cumple la desigualdad,

$$
(n-1) d t<r<n d t
$$

O sea que la distancia de $\boldsymbol{n d t}$ a r es infinitesimal. Esto quiere decir propiamente que la colección,

$$
\left\{t_{n}\right\}=\{n d t\}
$$

Determina un dominio continuo donde asume valores la variable $t=\left\{t_{n}\right\}$, ya que todo número real ordinario del intervalo $[-T, T]$ está infinitamente cerca de algún elemento del dominio dado por los valores $\left\{t_{n}\right\}$. Y esto es precisamente lo que de manera rigurosa significa la continuidad: el que dado $r \in[-T, T]$ se encuentra un $t$ tal que $r-t \approx 0$. 
Este enfoque difiere completamente del enfoque convencional sobre el continuo real, que sólo le atribuye tal propiedad al intervalo real $[-T, T]$.

El intervalo de muestreo o resolución en tiempo es el infinitesimal $d t$. Ya que la cantidad $f_{1}=\frac{1}{2 T}$ sigue siendo finita, tenemos así, los dominios asociados $\{n d t\} \Leftrightarrow\left\{k f_{1}\right\}$. Lo notorio ahora es que la variable temporal asume sus valores en el dominio continuo contenido en $[-T, T]$, mientras que la variable frecuencial sigue asumiendo valores en el dominio discreto, aunque la última frecuencia $\frac{N}{2 T}$ es un valor infinito, pues sobrepasa la recta real. Mientras no haya confusión, podríamos reescribir el par de dominios asociados en la forma $[-T, T] \Leftrightarrow\left\{k f_{0}\right\}$

Ello sugiere una asociación entre el dominio continuo limitado y el dominio discreto ilimitado.

De modo similar, vamos a ver qué ocurre en el dominio de la frecuencia, cuando se aumenta ilimitadamente, el período del tiempo.

\section{El continuo frecuencial}

¿Bajo qué condiciones el dominio discreto frecuencial puede convertirse en dominio continuo? O lo que es lo mismo, ¿bajo qué condiciones la frecuencia fundamental es infinitesimal? La frecuencia fundamental $f_{1}=\frac{1}{2 T}$ es finita porque la cantidad T es finita. De modo que será infinitesimal, si la cantidad $T$ se hace infinita. Veámoslo paso a paso.

Dupliquemos el intervalo limitado $[-T, T]$ y analicemos las alteraciones que se producen cuando el dominio temporal es $[-2 T, 2 T]$ y se cumple $-2 T \leq n<2 T$. Como el intervalo de muestreo temporal es el infinitesimal $d t=\frac{T}{N}$, para mantenerlo invariable, hemos duplicado el núme- ro de muestras. Ahora tenemos $4 \mathrm{~N}$ muestras, con intervalo de muestreo $\frac{2 T}{2 N}=\frac{T}{N}$ ¿ ¿Qué pasa con el dominio frecuencial? Obviamente, dicho dominio es $\left\{k f_{0}\right\}$, cuya más alta frecuencia es invariable, pues $\frac{2 N}{4 T}=\frac{N}{2 T}$. La duplicación del período ha conducido a que se intercalen $2 \mathrm{~N}$ frecuencias nuevas. Si triplicamos el período manteniendo fijo el intervalo de muestreo, se intercala el triple de frecuencias. Sea ahora $\boldsymbol{M}$ entero infinito. El intervalo $[-M T, M T]$ es infinito. El número de muestras temporales será $2 M N$, por lo que el intervalo de muestreo se mantiene en $\frac{M T}{M N}=\frac{T}{N}=d t$.

En el dominio de la frecuencia, hay un cambio radical. Como $M$ es entero infinito, la frecuencia fundamental $f_{1}=\frac{1}{2 M T}$ es infinitesimal!. Recordemos que el dominio frecuencial es la sucesión de valores

$f=\left\{\frac{k}{2 M T}\right\}=\left\{-\frac{M N}{2 M T},-\frac{M(N-1)}{2 M T}, \cdots,-\frac{2 M}{2 M T},-\frac{M}{2 M T}, 0, \frac{M}{2 M T}, \frac{2 M}{2 M T}, \cdots, \frac{M(N-1)}{2 M T}\right\}$

La diferencial es el valor constante $d f=\left\{\frac{1}{2 M T}\right\}$ y esta es precisamente la frecuencia fundamental $f_{1}$, que ahora es infinitesimal, por lo que $f$ se ha convertido en variable continua. Por tanto, la variable puede representarse como la sucesión $f=\{k d f\}$. El dominio frecuencial se ha convertido en un dominio continuo. Tanto el intervalo de muestreo $d t$ como la frecuencia fundamental $d f$ son infinitesimales. Los dominios asociados $\{n d t\} \Leftrightarrow\{k d f\}$ son ambos continuos.

\section{Conclusiones}

Resumimos nuestros resultados. Por el sólo hecho de considerar inmersas las variables y sus valores en un modelo hiperreal (donde se admiten infinitesimales y cantidades finitas e infinitas), los dominios temporales y frecuenciales discretos finitos se despliegan en tres partes correlacionadas: a) si $T$ es finito y $N$ finito, tanto el dominio temporal como el dominio frecuencial son dominios discretos finitos 


$$
\left\{\frac{n T}{N}\right\} \Leftrightarrow\left\{\frac{k}{2 T}\right\}
$$

b) Si $T$ es finito y $N$ entero infinito, el dominio temporal es continuo, mientras que el dominio frecuencial es discreto,

$$
\{n d t\} \Leftrightarrow\left\{\frac{k}{2 T}\right\}
$$

c) Si $M T$ es infinito y $d f=\frac{1}{2 M T}$, tanto el dominio temporal como el dominio frecuencial $\{n d t\} \Leftrightarrow\{k d f\}$ son dominios continuos.

En estos dominios se lleva a cabo la conversión de la Transformada Discreta de Fourier en Serie de Fourier, y de esta, en la Transformada Integral de Fourier.

\section{Referencias}

Bracewell, R. N. (1985). The Fourier Transform and its applications, 2a. Edición revisada McGraw-Hill Book Company, H. J. Weaver, (1983). Applications of discrete and Continuous Fourier Analysis, John Wiley\& Sons, New York,
Chichester, Brisbasne, Toronto, Sigapur.

Cochran, W. C. Cooley, J. W. et al ( 1967 ) What is the Fast Fourier Transform?, PROC., of the IEEE, Vol., 55, No. 10

George, K. (1998). Tesis Doctoral El cálculo discreto infinitesimal y la transformada de Fourier, CINVESTAV, México.

Luxemburg, W.A.J (1962) Non-standard Analysis. Lectures on A. Robinson's Theory of Infinitesimal and Infinitely Large Numbers, California Institute of Technology, Pasadena.

Pinsky, M. A. (2003) Introducción al análisis de Fourier y las ondeletas, MATH, Thomson.

Robinson A, Non-Standard (1966), Analysis, North-Holland Pub. Co, Amsterdam, Revised edition, 1974

Weaver, H. J. (1983) .Applications of discrete and Continuous Fourier Analysis, John Wiley\& Sons, New York, Chichester, Brisbasne, Toronto, Sigapur, 